\title{
Multicenter, phase II clinical trial of peptide vaccination with oral chemotherapy following curative resection for stage III colorectal cancer
}

\author{
JUNICHIRO KAWAMURA ${ }^{1}$, FUMIAKI SUGIURA ${ }^{2}$, YASUSHI SUKEGAWA ${ }^{3}$, YASUMASA YOSHIOKA ${ }^{1}$, \\ JIN-ICHI HIDA ${ }^{1}$, SHOICHI HAZAMA ${ }^{4,5}$ and KIYOTAKA OKUNO ${ }^{1}$
}

\author{
${ }^{1}$ Department of Surgery, Kindai University Faculty of Medicine, Osakasayama, Osaka 589-8511; ${ }^{2}$ Department of \\ Surgery, Seishukai Aiwa Hospital, Amagasaki, Hyougo 661-0953; ${ }^{3}$ Institute of Immunotherapy for Cancer, Kindai \\ University Faculty of Medicine, Osakasayama, Osaka 589-8511; ${ }^{4}$ Department of Gastroenterological, Breast and \\ Endocrine Surgery, Yamaguchi University Graduate School of Medicine; ${ }^{5}$ Department of Translational Research and \\ Developmental Therapeutics against Cancer, Yamaguchi University School of Medicine, Ube, Yamaguchi 755-8505, Japan
}

Received November 1, 2017; Accepted January 22, 2018

DOI: $10.3892 / 01.2018 .7905$

\begin{abstract}
The safety and immunological responsiveness of a peptide vaccine of ring finger protein 43 and $34-\mathrm{kDa}$ translocase of the outer mitochondrial membrane combined with uracil-tegafur/leucovorin (UFT/LV) was previously demonstrated in metastatic colorectal cancer (CRC) in a phase I clinical trial. To clarify the survival benefit of a peptide vaccine combined with UFT/LV as adjuvant treatment, a phase II clinical trial was conducted involving patients with stage III CRC. All enrolled patients, whose human leukocyte antigen (HLA)-A status was double-blinded, were administered the same regime of a peptide vaccine and UFT/LV chemotherapy. The primary objective of the study was to compare relapse-free survival (RFS) in patients with HLA-A*2402 vs. those without HLA-A*2402. Secondary objectives included comparisons between the two groups regarding overall survival, safety, tolerability and peptide-specific activities of cytotoxic T lymphocytes (CTLs) as measured by the ELISPOT assay. Between December 2009 and December 2014, a total of 46 patients were enrolled to the present study. Three-year RFS was not significantly different between HLA-A*2402 matched and unmatched groups [67.8 vs. $73.6 \%$, respectively; hazard ratio $(\mathrm{HR})=1.254,95 \%$ confidence interval $(\mathrm{CI})$ : $0.48-4.63 ; \mathrm{P}=0.706]$. Three-year RFS was significantly better in patients with positive CTL responses in the HLA-A*2402 matched group compared with those without (85.7 and $33.3 \%$, respectively; $\mathrm{HR}=0.159,95 \% \mathrm{CI}$ : $0.023-0.697 ; \mathrm{P}=0.011)$. In
\end{abstract}

Correspondence to: Professor Junichiro Kawamura, Department of Surgery, Kindai University Faculty of Medicine, 377-2 Ohno higashi, Osakasayama, Osaka 589-8511, Japan

E-mail: kawamuraj@med.kindai.ac.jp

Key words: colorectal cancer, clinical trial, peptide vaccine therapy, oral chemotherapy, and adjuvant chemotherapy conclusion, vaccination-induced immune responses combined with UFT/LV were positively associated with survival benefit in patients with HLA-A*2402-positive stage III CRC. Further study is required to clarify whether vaccination-induced immune responses shortly following the initiation of therapy can predict the therapeutic effect and help develop a promising therapeutic strategy for patients with stage III CRC.

\section{Introduction}

Colorectal cancer (CRC) represents the most common cause of cancer-related mortality and the second most common malignancy in Japan (1). Both the mortality rate and the prevalence of CRC have increased in Japan during recent decades (2), and around $30 \%$ of CRC patients have stage III disease. Postoperative adjuvant chemotherapy is systemic chemotherapy that is performed after surgery to prevent recurrence and improve the prognosis of patients who have undergone R0 resection (3). In general, patients with stage III CRC for whom $\mathrm{R} 0$ resection has been performed are indicated for adjuvant chemotherapy because of the relatively high recurrence rate for patients with stage III CRC (30.8\%) (4). 5-fluorouracil (FU)/leucovorin (LV) as adjuvant chemotherapy after surgery has prolonged survival for patients with advanced colon cancer compared with surgery alone (5). Moreover, the development of oral adjuvant chemotherapy such as uracil-tegafur (UFT)/LV (6), capecitabine (7), and S-1 (8) has further improved outcomes.

Chemotherapy of recurrent or metastatic CRC has improved substantially over the last 10 years through the development of new cytotoxic drugs including oxaliplatin (L-OHP) and irinotecan. Several studies in Western countries have demonstrated that the addition of L-OHP to FU/LV or capecitabine improved the adjuvant treatment of colon cancer (9-11). In Japan, however, some surgeons are skeptical of the use of L-OHP as adjuvant treatment for stage III colon cancer because the outcomes of Japanese randomized trials are better than those of Western countries. The ACTS-CC 
trial involving S-1 or UFT/LV reported a 3-year disease-free survival (DFS) rate using S-1 monotherapy for stage III colon cancer of $75.5 \%$, while the JCOG0205 trial involving 5-FU/LV vs UFT/LV reported a 3-year DFS rate using UFT/LV of $72.5 \%$; these results are comparable to those seen in trials in Europe and the US in which L-OHP was added to chemotherapy regimens $(7,8)$. Another disadvantage of L-OHP-based therapy is its cumulative neurotoxicity (12).

We previously reported a phase I clinical trial of a peptide vaccine ring finger protein 43 (RNF43) and 34-kDa translocase of the outer mitochondrial membrane (TOMM34) combined with UFT/LV for metastatic CRC, and demonstrated the safety and immunological responsiveness of this combination therapy (13). To clarify the survival benefit of a peptide vaccine combined with UFT/LV as adjuvant treatment, we conducted a multicenter, phase II clinical trial of patients with stage III CRC.

\section{Materials and methods}

Patients and eligible criteria. Patients were eligible for enrollment if they were 20-80 years old with histologically confirmed stage III CRC, had adequate critical organ functions, and had an Eastern Cooperative Oncology Group performance status of 0 or 1 . Patients were excluded if they were pregnant, breastfeeding, were trying to become pregnant, had an active infectious disease, had multiple cancers, or took steroids or immunosuppressive therapy. The study was carried out in accordance with the Declaration of Helsinki on experimentation with human subjects, and was approved by Institutional Ethical Review Boards of Kindai University (approval no. 20-110) and of Yamaguchi University School of Medicine (approval no. H22-175). It was registered at the UMIN Clinical Trials Registry as UMIN 000003552 (http://www.umin. ac.jp/ctr/index.htm). Written informed consent was obtained from all patients at the time of enrollment.

Peptides and drugs. HLA-A*2402-restricted RNF43 (NSQPVWLCL) and TOMM34 (KLRQEVKQNL) peptides were synthesized by American Peptide Company Inc. (Sunnyvale, CA, USA) according to a standard solid-phase synthesis method; preclinical trials previously confirmed that the peptides did not produce acute toxicity (13).

Montanide ISA-51 (also known as incomplete Freund's adjuvant) is a sterile vaccine adjuvant manufactured by SEPPIC Co. (Puteaux, France) in accordance with good manufacturing practice standards. Montanide is currently used as an adjuvant in vaccine therapies worldwide, and no serious adverse events caused by Montanide have been reported.

UFT is a relatively old oral fluoropyrimidine that was developed in Japan in the 1980s. It has many indications for metastatic and advanced solid cancers including those of the colon, lung, breast, and pancreas, and gastric cancer (14). In metastatic CRC, UFT/LV was demonstrated to have the same clinical efficacy as 5-FU/LV and comparable pharmacokinetics between Japanese and American patients (15-17). We previously demonstrated that the standard dose of UFT/LV did not impede the immune responses of patients with advanced CRC to peptides administered as cancer vaccination (18)
Study design. This phase II, non-randomized, single arm study in which the HLA-A status was double-blinded aimed to clarify the survival benefit of a peptide vaccine in combination with UFT/LV as adjuvant treatment for patients with stage III CRC. The therapy consisted of a cocktail of two epitope peptides with UFT/LV. Although the peptides used in this study were HLA-A*2402-restricted, all enrolled patients whose HLA-A status was double-blinded were administered the same regime of peptide cocktail and UFT/LV chemotherapy. The cocktail of two peptides (1 mg of each peptide) was mixed with Montanide ISA 51 and subcutaneously administered to patients once every 7 days five times. All patients also received daily oral doses of UFT (300 mg/m²/day) plus LV (UZEL: $75 \mathrm{mg} /$ day) for 28 days. Each cycle of treatment was followed by 1 week of rest. Patients received six cycles of treatment unless their disease relapsed.

Study objectives. The primary objective was the comparison of RFS between patients with HLA-A*2402 vs. those without HLA-A*2402. Secondary objectives included comparisons between the two groups regarding overall survival (OS), safety, tolerability, and peptide-specific activities of cytotoxic Tlymphocytes (CTLs). Adverse events resulting from the peptide vaccine were evaluated using the National Cancer Institute's Common Terminology Criteria for Adverse Events v.4.0 (19).

ELISPOT assay. Peptide-specific CTL responses were estimated by the in vitro ELISPOT assay as previously described (20). Briefly, frozen peripheral blood mononuclear cells (PBMCs) from each patient were thawed simultaneously. PBMCs $\left(5 \times 10^{5} / \mathrm{ml}\right)$ were then cultured with $10 \mathrm{microgram} / \mathrm{ml}$ of each peptide and $100 \mathrm{IU} / \mathrm{ml}$ of interleukin-2 (Novartis, Emeryville, CA, USA) at $37^{\circ} \mathrm{C}$ for 2 weeks. Peptides were added to the cultures on day 0 and day 7 . Following CD4+ cell depletion by the Dynal CD4 positive isolation kit (Invitrogen, Carlsbad, CA, USA), interferon (IFN)- $\gamma$ ELISPOT assays were performed using the Human IFN- $\gamma$ ELISpot PLUS kit (MabTech, Nacka Strand, Sweden), according to the manufacturer's instructions. The positivity of antigen-specific $\mathrm{T}$ cell response was quantitatively defined according to the evaluation tree algorithm described by Kono et al (21). Briefly, the peptide-specific T cell responses were classified into four grades $(-,+,++$, and $+++)$ depending on the peptide-specific spots at different responder/stimulator ratios. We judged to be positive case, when the algorithm indicated,+++ , or +++ .

Statistical analyses. This study defined the HLA-A*2402 matched group as the study group and the unmatched group as the control group. We estimated that a minimum of 42 patients would be required for the HLA-A*2402 unmatched group and 64 for the HLA-A*2402 matched group, assuming a RFS of $75 \%$ in the HLA unmatched control group and that of $85 \%$ in the HLA matched study group with a two-sided $\alpha$ level of 0.05 and a beta level of 0.2 . Considering the distribution (approximately 67\%) of HLA-A*2402 in the Japanese population and some dropout cases, we decided to enroll a total of 110 patients.

Qualitative data were reported as the number of patients, and were compared using either Pearson's $\chi^{2}$ test or Fisher's exact test, as appropriate. The Wilcoxon rank sum test was used to compare qualitative data. Survival curves were plotted using the Kaplan-Meier method and compared with the log-rank test. 
Table I. Patient background.

\begin{tabular}{|c|c|c|c|c|}
\hline Characteristic & Total $(n=44)$ & HLA-A*2402 $(n=28)$ & non-HLA-A*2402 (n=16) & P-value \\
\hline Age, median (range), years & $64(37-80)$ & $63(37-76)$ & $64(47-80)$ & n.s. \\
\hline \multicolumn{5}{|l|}{ Sex, n $(\%)$} \\
\hline Male & $20(45 \%)$ & $14(50 \%)$ & $6(37 \%)$ & \multirow[t]{2}{*}{ n.s. } \\
\hline Female & $24(55 \%)$ & $14(50 \%)$ & $10(63 \%)$ & \\
\hline Number of vaccination, median (range) & $30(21-30)$ & $30(21-30)$ & $30(30-30)$ & n.s. \\
\hline \multicolumn{5}{|l|}{ Colon/rectum, n (\%) } \\
\hline Colon & $25(57 \%)$ & $17(61 \%)$ & $7(43 \%)$ & \multirow[t]{2}{*}{ n.s. } \\
\hline Rectum & $19(43 \%)$ & $11(39 \%)$ & $9(57 \%)$ & \\
\hline \multicolumn{5}{|l|}{ Location of primary tumor, $\mathrm{n}(\%)$} \\
\hline Right & $9(20 \%)$ & $7(25 \%)$ & $5(31 \%)$ & \multirow[t]{2}{*}{ n.s. } \\
\hline Left & $26(60 \%)$ & $21(75 \%)$ & $10(69 \%)$ & \\
\hline \multicolumn{5}{|l|}{ Stage, n (\%) } \\
\hline IIIa & $28(64 \%)$ & $15(54 \%)$ & $13(81 \%)$ & \multirow[t]{2}{*}{ n.s. } \\
\hline $\mathrm{IIIb}$ & $16(36 \%)$ & $13(46 \%)$ & $3(19 \%)$ & \\
\hline
\end{tabular}

HLA-A, human leukocyte antigen-A; n.s., not significant.

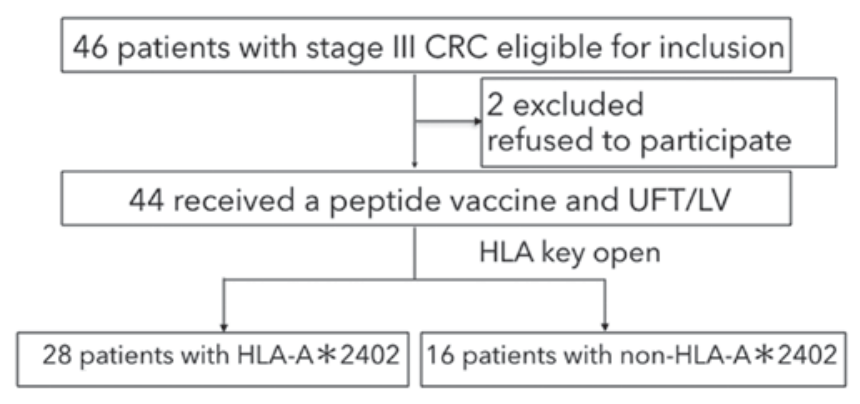

Figure 1. Flow diagram of patient grouping and recruitment. CRC, colorectal cancer; HLA-A, human leukocyte antigen-A; UFT/LV, uracil-tegafur/leucovorin.

Survival was measured from the first vaccination until recurrence, death, or the last follow-up. Tests were always two-sided and the level of statistical significance was set at $\mathrm{P}<0.05$. Statistical analysis was performed using JMP 11 software (SAS Institute Inc., Cary, NC, USA).

\section{Results}

Demographics. The patient flow diagram is shown in Fig. 1. Between December 2009 and December 2014, a total of 46 patients were enrolled in the study. Although we planned to recruit 110 patients, the registration was prematurely closed because of slow patient recruitment. This might reflect the fact that adjuvant chemotherapy with oxaliplatin-based regimens were approved in August 2009, so patients may have wished to receive an oxaliplatin-based regimen rather than an uncertain peptide vaccine treatment. Moreover, approximately $40 \%$ of patients were predicted to have no benefit with the peptide vaccine treatment.

Twenty-eight patients had at least one allele of HLA-A*2402 and 16 had no HLA-A*2402 allele. Among the 46 patients,
44 received peptide vaccine therapy with UFT/LV (Fig. 1). Two patients were excluded because they withdrew consent. As shown in Table I, there was no significant difference between the HLA-A*2402 matched and unmatched groups regarding gender, age, location of the primary tumor, dose of vaccine peptides administered, and the number of positive lymph node metastases (i.e., $\leqq 3$ vs. $>3$ ) which were synonymous with stage IIIa vs. stage IIIb based on the Japanese Classification of Colorectal Cancer (22). Fewer patients with stage IIIb were in the HLA-A*2402 unmatched group than the HLA-A*2402 matched group, but this difference was not significant $(\mathrm{P}=0.059)$.

Survival and recurrence. The median duration of follow-up for the overall study population was 54 (range, 11-88) months. There was no significant difference between HLA-A*2402 matched and unmatched groups regarding the 3 -year RFS (67.8 vs. $73.6 \%$, respectively, hazard ratio $(\mathrm{HR})=1.254,95 \%$ confidence interval (CI): 0.48-4.63, $\mathrm{P}=0.706$ ) (Fig. 2A)., nor regarding $\mathrm{OS}(\mathrm{HR}=1.40$, 95\% CI: 0.30-9.84, $\mathrm{P}=0.683$ ) (Fig. 2B).

Subgroup analysis. To minimize the potential bias regarding fewer stage IIIb CRC patients in the HLA-A*2402 unmatched group, subgroup analysis was conducted for each stage IIIa or stage IIIb group. No significant difference was observed within stage IIIa or stage IIIb regarding gender, age, location of the primary tumor, or the dose of vaccine peptides administered (Table IIA and B). There was also no significant difference between HLA-A*2402 matched and unmatched stage IIIa CRC groups regarding 3-year RFS (86.7 and 91.7\%, respectively, $\mathrm{HR}=1.63$, 95\% CI: 0.156-35.0, P=0.687) (Fig. 3). 3-year OS was $100 \%$ in both HLA-A*2402 matched and unmatched stage IIIa CRC groups.

The 3-year RFS in HLA-A*2402 matched and unmatched stage IIIb CRC groups was 46.1 and $0 \%$, respectively $(\mathrm{HR}=0.293,95 \%$ CI: $0.070-1.45, \mathrm{P}=0.0789)$, with the 
Table II. Characteristics of patients with stage IIIa and IIIb colorectal cancer.

A, Stage IIIa colorectal cancer

\begin{tabular}{|c|c|c|c|c|}
\hline Variable & $\begin{array}{l}\text { Total number of } \\
\text { patients }(n=28)\end{array}$ & $\begin{array}{l}\text { HLA-A*2402 } \\
\quad(n=15)\end{array}$ & $\begin{array}{c}\text { non-HLA-A } * 2402 \\
2402(n=13)\end{array}$ & P-value \\
\hline Age, median (range), years & $64(37-80)$ & $66(37-74)$ & $63(50-80)$ & n.s. \\
\hline Sex, n $(\%)$ & & & & n.s. \\
\hline Male & $12(43 \%)$ & $7(47 \%)$ & $5(38 \%)$ & \\
\hline Female & $16(57 \%)$ & $8(53 \%)$ & $8(62 \%)$ & \\
\hline Number of vaccination, median (range) & $30(30-30)$ & $30(30-30)$ & $30(30-30)$ & n.s. \\
\hline Colon/rectum, n (\%) & & & & n.s. \\
\hline Colon & $18(64 \%)$ & $12(80 \%)$ & $6(46 \%)$ & \\
\hline Rectum & $10(36 \%)$ & $3(20 \%)$ & $7(54 \%)$ & \\
\hline Location of primary tumor, n (\%) & & & & n.s. \\
\hline Right & $8(29 \%)$ & $4(27 \%)$ & $4(31 \%)$ & \\
\hline Left & $20(71 \%)$ & $11(73 \%)$ & $9(69 \%)$ & \\
\hline
\end{tabular}

B, Stage IIIb colorectal cancer

\begin{tabular}{|c|c|c|c|c|}
\hline Variable & $\begin{array}{l}\text { Total number of } \\
\text { patients }(n=28)\end{array}$ & $\begin{array}{l}\text { HLA-A*2402 non- } \\
\qquad(n=15)\end{array}$ & $\begin{array}{l}2402 \\
2402(n=13)\end{array}$ & P-value \\
\hline Age, median (range), years & $61(39-80)$ & $60(39-76)$ & $80(47-80)$ & n.s. \\
\hline Sex, n $(\%)$ & & & & n.s. \\
\hline Male & $8(50 \%)$ & $7(54 \%)$ & $1(33 \%)$ & \\
\hline Female & $8(50 \%)$ & $6(46 \%)$ & $2(67 \%)$ & \\
\hline Number of vaccination, median (range) & $30(21-30)$ & $30(21-30)$ & $30(30-30)$ & n.s. \\
\hline Colon/rectum, n (\%) & & & & n.s. \\
\hline Colon & $6(38 \%)$ & $5(38 \%)$ & $1(33 \%)$ & \\
\hline Rectum & $10(62 \%)$ & $8(62 \%)$ & $2(67 \%)$ & \\
\hline Location of primary tumor, $\mathrm{n}(\%)$ & & & & n.s. \\
\hline Right & $4(25 \%)$ & $3(23 \%)$ & $1(33 \%)$ & \\
\hline Left & $12(75 \%)$ & $10(77 \%)$ & $2(67 \%)$ & \\
\hline
\end{tabular}

HLA-A, human leukocyte antigen-A; n.s., not significant.

HLA-A*2402 matched stage IIIb group showing a nonsignificant trend toward better survival (Fig. 4). There was no significant difference in OS between HLA-A*2402 matched and unmatched stage IIIb CRC groups.

Safety. The most frequent adverse event was vaccination-site reaction $(n=39)$, and all of the events were grade 1 or 2 . The vaccination therapy was well-tolerated with no treatment-associated adverse events $\geq$ grade 3 , except for two cases. One patient had grade 3 transaminase elevation, which recovered after cessation of the drug. However, after 28 vaccine peptide administrations, the recurrence of peritoneal dissemination was detected so the adjuvant therapy was discontinued (Table III). Another patient developed bowel perforation after 21 administrations of the vaccine peptide, so the adjuvant therapy was discontinued, though a causal relationship with the adverse event was not proven.
Immunological evaluation in the HLA-A*2402 matched group. Peptide-specific CTL responses were estimated by the in vitro ELISPOT assay before the initiation of therapy and after two cycles of treatment. In the HLA-A*2402 matched group, positive or negative CTL responses specific for the RNF43 and/or TOMM34 peptides after two cycles of treatment were observed in 14 and nine patients, respectively; the CTL responses of five patients were not detected (Table IV). The 3-year RFS was significantly better in patients with positive CTL responses than in those without in the HLA-A*2402 matched group (85.7 vs. $33.3 \%$, respectively, $H R=0.159$, 95\% CI: 0.023-0.697, $\mathrm{P}=0.011$ ) (Fig. 5).

\section{Discussion}

This phase II clinical trial demonstrated that vaccination-induced immune responses combined with UFT/LV are 
Table III. Number of adverse events in patients.

\begin{tabular}{|c|c|c|c|c|c|}
\hline Toxicity & Total n (\%) & Grade 1 & Grade 2 & Grade 3 & Grade 4 \\
\hline Anemia & $3(7)$ & $3(7 \%)$ & 0 & 0 & 0 \\
\hline Neutrophil count decreased & $3(7)$ & $2(4 \%)$ & $1(2 \%)$ & 0 & 0 \\
\hline Transaminase elevation & $9(20)$ & $8(18 \%)$ & 0 & $1(2 \%)$ & 0 \\
\hline Hyperbilirubinemia & $4(9)$ & $4(9 \%)$ & 0 & 0 & 0 \\
\hline Anorexia & $6(14)$ & $6(14 \%)$ & 0 & 0 & 0 \\
\hline Malaise & $5(11)$ & $5(11 \%)$ & 0 & 0 & 0 \\
\hline Diarrhea & $8(18)$ & $5(11 \%)$ & $3(7 \%)$ & 0 & 0 \\
\hline Hand-foot skin reaction & $5(11)$ & $5(11 \%)$ & 0 & 0 & 0 \\
\hline Sensory neuropathy & $2(4)$ & $2(4 \%)$ & 0 & 0 & 0 \\
\hline Skin hyperpigmentation & $3(12)$ & $3(12 \%)$ & 0 & 0 & 0 \\
\hline Stomatitis & $4(9)$ & $4(9 \%)$ & 0 & 0 & 0 \\
\hline Colonic perforation & $1(2)$ & 0 & 0 & 0 & $1(2 \%)$ \\
\hline Vaccination site reaction & $39(88)$ & $31(70 \%)$ & $8(18 \%)$ & 0 & 0 \\
\hline
\end{tabular}
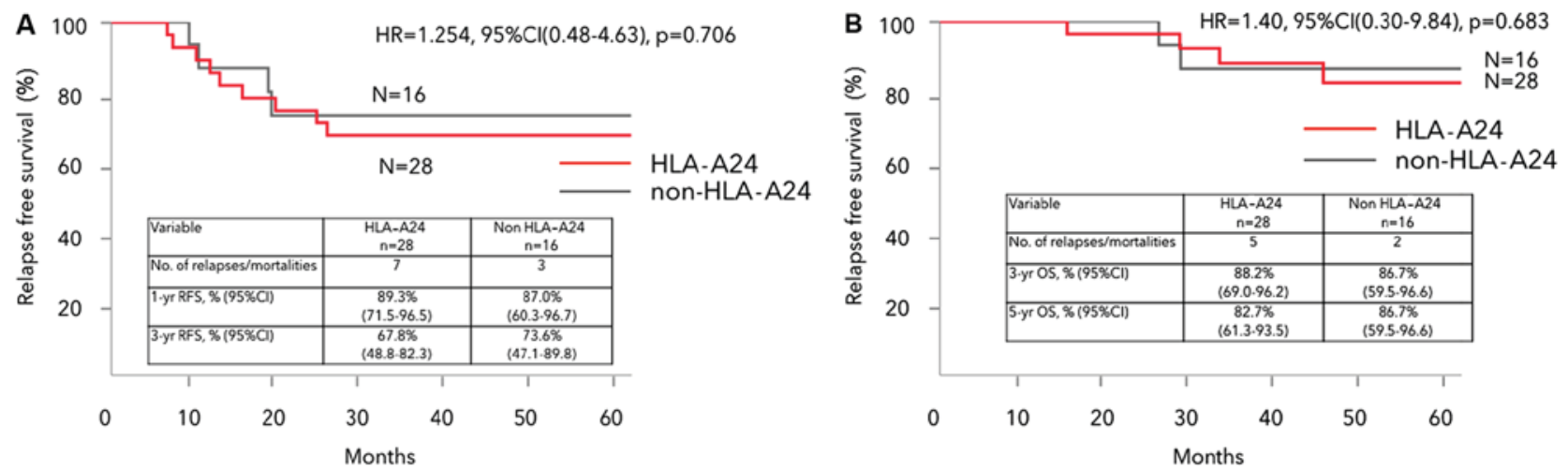

Figure 2. Kaplan-Meier analysis of (A) RFS and (B) OS in HLA-A*2402 matched and unmatched groups. RFS and OS showed no significant difference between the HLA-A*2402 matched and unmatched groups. RFS, relapse-free survival; HLA-A, human leukocyte antigen-A; HR, hazard ratio; CI, confidence interval; OS, overall survival.

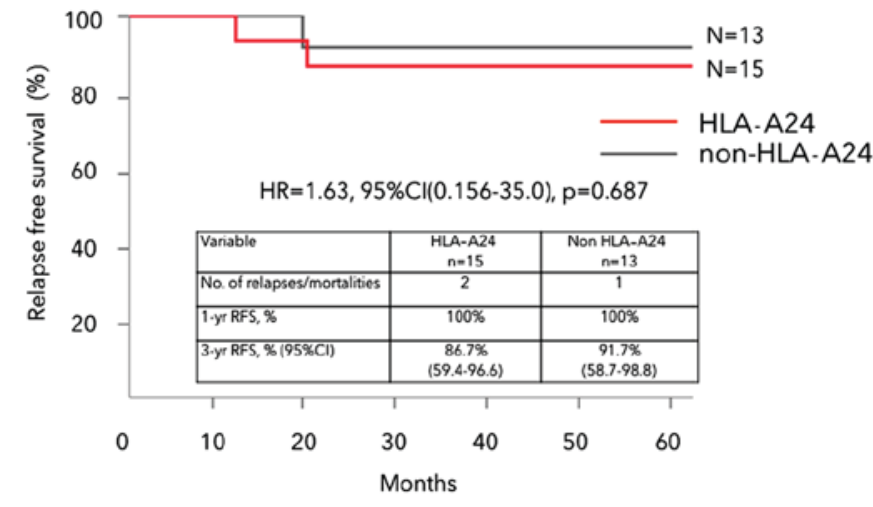

Figure 3. Kaplan-Meier analysis of RFS in HLA-A*2402 matched and unmatched stage IIIa CRC groups. RFS showed no significant difference between the HLA-A*2402 matched and unmatched stage IIIa CRC groups. RFS, relapse-free survival; HLA-A, human leukocyte antigen-A; HR, hazard ratio; $\mathrm{CI}$, confidence interval; $\mathrm{CRC}$, colorectal cancer.

positively associated with survival benefit in patients with HLA-A*2402-positive stage III CRC. Importantly, none of the patients in the stage IIIa group with positive CTL responses

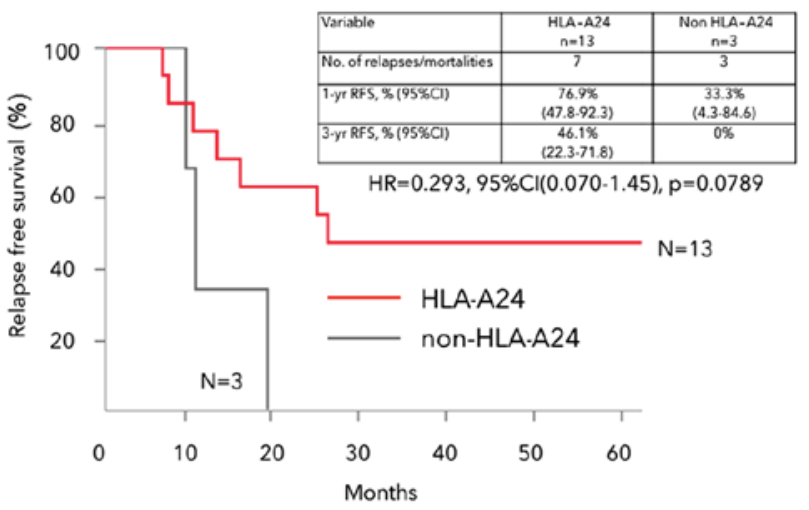

Figure 4. Kaplan-Meier analysis of RFS in HLA-A*2402 matched and unmatched stage IIIb CRC groups. The HLA-A*2402 matched stage IIIb group had a nonsignificant trend toward better survival. RFS, relapse-free survival; HLA-A, human leukocyte antigen-A; HR, hazard ratio; CI, confidence interval; $\mathrm{CRC}$, colorectal cancer.

specific for RNF43 and/or TOMM34 peptides after two cycles of treatment relapsed at all, while two out of three patients with negative CTL responses did relapse (data not shown). In 
Table IV. In vitro ELISPOT assay prior to the initiation of therapy and following 2 cycles of treatment in the human leukocyte antigen-A2402 matched group.

CTL response (RNF43/TOMM34)

\begin{tabular}{|c|c|c|}
\hline Patient no. & $\begin{array}{l}\text { Prior to the } \\
\text { initiation of therapy }\end{array}$ & $\begin{array}{c}\text { Following } 2 \\
\text { cycles of therapy }\end{array}$ \\
\hline 1 & $+/+$ & $\mathrm{NA} / \mathrm{NA}^{\mathrm{a}}$ \\
\hline 2 & $-/-$ & $-/ \mathrm{NA}^{\mathrm{a}}$ \\
\hline 3 & $-/+$ & $-/+$ \\
\hline 4 & $-/-$ & $+/-$ \\
\hline 5 & $-/+$ & $-/-$ \\
\hline 6 & $-/-$ & $\mathrm{NA} / \mathrm{NA}^{\mathrm{a}}$ \\
\hline 7 & $+/-$ & $+/-$ \\
\hline 8 & $-/-$ & $+/-$ \\
\hline 9 & $-/-$ & $-/+$ \\
\hline 10 & $-/-$ & $-/-$ \\
\hline 11 & $-/-$ & $+/-$ \\
\hline 12 & $-/-$ & $-/-$ \\
\hline 13 & $+/-$ & $+/-$ \\
\hline 14 & $+/-$ & $-/+$ \\
\hline 15 & $-/-$ & $-/-$ \\
\hline 16 & $-/-$ & $+/-$ \\
\hline 17 & $+/ \mathrm{NA}$ & $+/+$ \\
\hline 18 & -/NA & $-/-$ \\
\hline 19 & $-/-$ & $-/-$ \\
\hline 20 & $-/-$ & $-/-$ \\
\hline 21 & $-/-$ & $-/-$ \\
\hline 22 & $+/+$ & $-/ \mathrm{NA}^{\mathrm{a}}$ \\
\hline 23 & $-/-$ & $+/ \mathrm{NA}$ \\
\hline 24 & -/NA & $-/-$ \\
\hline 25 & $-/-$ & $-/+$ \\
\hline 26 & $+/-$ & $+/-$ \\
\hline 27 & $+/-$ & $-/+$ \\
\hline 28 & $-/-$ & $-/ \mathrm{NA}^{\mathrm{a}}$ \\
\hline
\end{tabular}

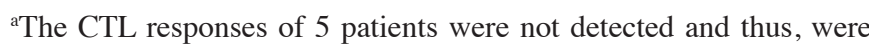
excluded from the analysis. NA, not available; CTL, cytotoxic T lymphocyte; RNF43, ring finger protein 43; TOMM34, 34-kDa translocase of the outer mitochondrial membrane.

our earlier trial of the same treatment strategy, no remarkable clinical responses were observed in patients with metastatic CRC refractory to standard chemotherapy (13). However, in this trial, the clear advantage of positive CTL responses was observed as an adjuvant setting for CRC patients with lymph node metastasis after curative resection; thus, peptide vaccination is likely to be more effective for low-risk CRC patients than high-risk or metastatic CRC patients.

In this trial, two patients developed grade 3 or higher adverse events, although these were not proven to have a causal relationship with the peptide vaccination combined with UFT/LV. Moreover, the vaccination therapy was otherwise well-tolerated with no other treatment-associated adverse

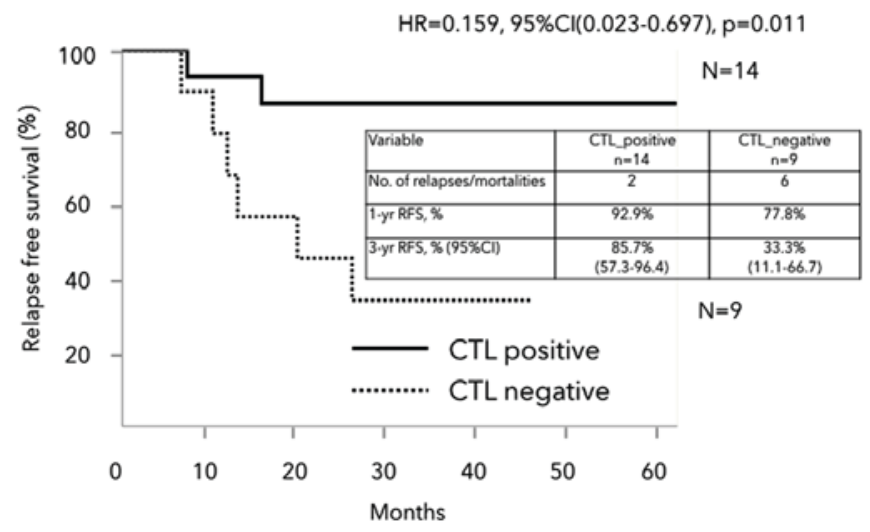

Figure 5. Kaplan-Meier analysis of RFS in the HLA-A*2402 matched group. Patients with positive CTL responses had a significantly better RFS than those without (HR=0.159, 95\% CI: 0.023-0.697, $\mathrm{P}=0.011$ ). RFS, relapse-free survival; HLA-A, human leukocyte antigen-A; HR, hazard ratio; CI, confidence interval; CRC, colorectal cancer; CTL, cytotoxic T lymphocyte.

events of grade 3 or higher. With the exception of the vaccication site reaction, the rates of other adverse events did not exceed those of UFT/LV chemotherapy, and the addition of the peptide vaccination did not increase the adverse events, which was the same as that reported in our previous trial (13).

Peptide-specific CTL responses were estimated by the in vitro ELISPOT assay before the initiation of therapy and after two cycles of treatment. In the HLA-A*2402 matched group, patients with positive CTL responses after two cycles of treatment showed a significantly better survival than those without. Peptide-specific CTL responses were also observed in HLA-A24 unmatched group, and patients with positive CTL responses in this group had a nonsignificant improvement in prognosis than those with negative CTL responses (data not shown). These peptides used in this study had been considered HLA-A*2402-restricted, however, the possible cross reactivity of the peptides to other serotypes should be taken into account. Since the affinity of the peptides-HLA-A24 should be higher than that of the peptides-other serotypes, the difference of the affinity might influence the clinical outcomes. Vaccination-induced immune responses are positively associated with survival benefit in HLA-A*2402-positive group, and vaccination-induced immune responses that occur shortly after the initiation of therapy could be used to predict the therapeutic effect.

This study has a number of limitations. First, its sample size was small. Therefore, to confirm the survival benefit of peptide vaccination with UFT/LV for patients with HLA-A*2402-positive stage III CRC, additional cases should be recruited to achieve adequate statistical power because some patients lacked positive CTL responses specific for RNF43 and/or TOMM34 peptides. Additionally, the background of the patients was biased. The HLA-A*2402-positive stage IIIb group only contained three patients, of whom most had rectal cancers. Thus, the outcomes of these patients were worse than expected. Second, although the HLA-A*2402 matched stage IIIb group had a nonsignificant trend toward better survival than the HLA-A*2402 unmatched stage IIIb group, its 3-year RFS was $46.1 \%$, which was inferior to that seen in the JCOG0205 trial. While the stage IIIb group in 
this study was supposed to have a worse survival because of the high proportion of rectal cancer patients, we found no evidence to support the validity of an L-OHP-free regimen as adjuvant chemotherapy. Third, this study did not compare outcomes between patients receiving peptide vaccination combined with UFT/LV vs. those who did not. In assessing the value of the peptide vaccination, an appropriate control would be HLA-A*2402-positive CRC patients who received UFT/LV with no peptide vaccination.

In conclusion, vaccination-induced immune responses combined with UFT/LV are positively associated with survival benefit in patients with HLA-A*2402-positive stage III CRC. Further study is needed to clarify whether vaccination-induced immune responses that occur shortly after the initiation of therapy can be used to predict the therapeutic effect and help develop a therapeutic strategy.

\section{Acknowledgements}

This study was supported partially by JSPS KAKENHI (grant no. 15K10153). We greatly appreciate the excellent advice and cooperation of Dr. Sachiko Yoshimura from OncoTherapy Science, Inc. and Professor Yusuke Nakamura at the University of Chicago, who also provided all the peptides used in this study. The authors would like to thank Dr Sarah Williams for editing a draft of this manuscript.

\section{References}

1. Data from Cancer Registry and Statistics. Cancer Information Service, National Cancer Center, Japan. https://ganjoho.jp/ reg_stat/statistics/dl/index.html\#incidence. Accessed October 24, 2017 (In Japanese).

2. Vital Statistics. Ministry of Health, Labour and Welfare, Japan. http://www.mhlw.go.jp/toukei/list/81-1.html. Accessed October 24, 2017 (In Japanese).

3. NIH consensus conference. Adjuvant therapy for patients with colon and rectal cancer. JAMA 264: 1444-1450, 1990.

4. Watanabe T, Itabashi M, Shimada Y, Tanaka S, Ito Y, Ajioka Y, Hamaguchi T, Hyodo I, Igarashi M, Ishida $\mathrm{H}$, et al: Japanese society for cancer of the colon and rectum (JSCCR) guidelines 2014 for treatment of colorectal cancer. Int J Clin Oncol 20: 207-239, 2015.

5. Wolmark N, Rockette H, Fisher B, Wickerham DL, Redmond C, Fisher ER, Jones J, Mamounas EP, Ore L and Petrelli NJ: The benefit of leucovorin-modulated fluorouracil as postoperative adjuvant therapy for primary colon cancer: Results from national surgical adjuvant breast and bowel project protocol C-03. J Clin Oncol 11: 1879-1887, 1993.

6. Shimada Y, Hamaguchi T, Mizusawa J, Saito N, Kanemitsu Y, Takiguchi N, Ohue M, Kato T, Takii Y, Sato T, et al: Randomised phase III trial of adjuvant chemotherapy with oral uracil and tegafur plus leucovorin versus intravenous fluorouracil and levofolinate in patients with stage III colorectal cancer who have undergone Japanese D2/D3 lymph node dissection: Final results of JCOG0205. Eur J Cancer 50: 2231-2240, 2014.

7. Twelves C, Wong A, Nowacki MP, Abt M, Burris H III, Carrato A, Cassidy J, Cervantes A, Fagerberg J, Georgoulias V, et al: Capecitabine as adjuvant treatment for stage III colon cancer. N Engl J Med 352: 2696-2704, 2005.

8. Yoshida M, Ishiguro M, Ikejiri K, Mochizuki I, Nakamoto Y Kinugasa Y, Takagane A, Endo T, Shinozaki H, Takii Y, et al: S-1 as adjuvant chemotherapy for stage III colon cancer: A randomized phase III study (ACTS-CC trial). Ann Oncol 25: 1743-1749, 2014.
9. André T, Boni C, Mounedji-Boudiaf L, Navarro M, Tabernero J, Hickish T, Topham C, Zaninelli M, Clingan P, Bridgewater J, et al: Oxaliplatin, fluorouracil, and leucovorin as adjuvant treatment for colon cancer. N Engl J Med 350: 2343-2351, 2004.

10. Kuebler JP, Wieand HS, O'Connell MJ, Smith RE, Colangelo LH, Yothers G, Petrelli NJ, Findlay MP, Seay TE, Atkins JN, et al: Oxaliplatin combined with weekly bolus fluorouracil and leucovorin as surgical adjuvant chemotherapy for stage II and III colon cancer: Results from NSABP C-07. J Clin Oncol 25: 2198-2204, 2007.

11. Schmoll HJ, Tabernero J, Maroun J, de Braud F, Price T, Van Cutsem E, Hill M, Hoersch S, Rittweger K and Haller DG: Capecitabine plus oxaliplatin compared with fluorouracil/folinic acid as adjuvant therapy for stage III colon cancer: Final results of the NO16968 randomized controlled phase III trial. J Clin Oncol 33: 3733-3740, 2015.

12. André T, Boni C, Navarro M, Tabernero J, Hickish T, Topham C, Bonetti A, Clingan P, Bridgewater J, Rivera F and de Gramont A: Improved overall survival with oxaliplatin, fluorouracil, and leucovorin as adjuvant treatment in stage II or III colon cancer in the MOSAIC trial. J Clin Oncol 27: 3109-3116, 2009.

13. Okuno K, Sugiura F, Hida JI, Tokoro T, Ishimaru E, Sukegawa Y and Ueda K: Phase I clinical trial of a novel peptide vaccine in combination with UFT/LV for metastatic colorectal cancer. Exp Ther Med 2: 73-79, 2011.

14. Tanaka F: UFT (tegafur and uracil) as postoperative adjuvant chemotherapy for solid tumors (carcinoma of the lung, stomach, colon/rectum and breast): Clinical evidence, mechanism of action, and future direction. Surg Today 37: 923-943, 2007.

15. Carmichael J, Popiela T, Radstone D, Falk S, Borner M, Oza A, Skovsgaard T, Munier S and Martin C: Randomized comparative study of tegafur/uracil and oral leucovorin versus parenteral fluorouracil and leucovorin in patients with previously untreated metastatic colorectal cancer. J Clin Oncol 20: 3617-3627, 2002.

16. Douillard JY, Hoff PM, Skillings JR, Eisenberg P, Davidson N, Harper P, Vincent MD, Lembersky BC, Thompson S, Maniero A and Benner SE: Multicenter phase III study of uracil/tegafur and oral leucovorin versus fluorouracil and leucovorin in patients with previously untreated metastatic colorectal cancer. J Clin Oncol 20: 3605-3616, 2002.

17. Shirao K, Hoff PM, Ohtsu A, Loehrer PJ, Hyodo I, Wadler S, Wadleigh RG, O'Dwyer PJ, Muro K, Yamada Y, et al: Comparison of the efficacy, toxicity, and pharmacokinetics of a uracil/tegafur (UFT) plus oral leucovorin (LV) regimen between Japanese and American patients with advanced colorectal cancer: Joint United States and Japan study of UFT/LV. J Clin Oncol 22: 3466-3474, 2004.

18. Hattori T, Mine T, Komatsu N, Yamada A, Itoh K, Shiozaki H and Okuno K: Immunological evaluation of personalized peptide vaccination in combination with UFT and UZEL for metastatic colorectal carcinoma patients. Cancer Immunol Immunother 58: $1843-1852,2009$.

19. U.S. Department of Health and Human Services, National Institutes of Health and National Cancer Institute: Common Terminology Criteria for Adverse Events (CTCAE). Version4.0. https://evs.nci.nih.gov/ftp1/CTCAE/CTCAE 4.03_2010-06-14 QuickReference_5x7.pdf. Accessed May, 2009.

20. Okuno K, Sugiura F, Inoue K and Sukegawa Y: Clinical trial of a 7-peptide cocktail vaccine with oral chemotherapy for patients with metastatic colorectal cancer. Anticancer Res 34: 3045-3052, 2014.

21. Kono K, Iinuma H, Akutsu Y, Tanaka H, Hayashi N, Uchikado Y, Noguchi T, Fujii H, Okinaka K, Fukushima R, et al: Multicenter, phase II clinical trial of cancer vaccination for advanced esophageal cancer with three peptides derived from novel cancer-testis antigens. J Transl Med 10: 141, 2012.

22. Japanese Society for Cancer of the Colon anc Rectum: Japanese Classification of Colorectal Carcinoma. 8th edition. Kanehara \& Co., Ltd., Tokyo, 2013.

This work is licensed under a Creative Commons Attribution-NonCommercial-NoDerivatives 4.0 International (CC BY-NC-ND 4.0) License. 